MAX-PLANCK-INSTITUT FÜR W ISSENSCHAFTSGESCHICHTE

Max Planck Institute for the History of Science

PREPRINT $2\left(\begin{array}{lll}19 & 9 & 4\end{array}\right)$

J ürgen Renn

Historical Epistemology and Interdisciplinarity 


\title{
Historical Epistemology and Interdisciplinarity
}

\author{
The basic ideology of science is mastery of \\ nature, a basic component of modern society. \\ But that mastery must be mastered, by \\ philosophy and politics, which is to say, by \\ insight, activity and change. \\ Robert S. Cohen ${ }^{1}$
}

\section{Jürgen Renn}

The subject of interdisciplinarity concerns the philosophy and the history of science in a twofold way: on the one hand the emergence, the development, and the mutual relationships between disciplines are, of course, one of the central subjects of research in the history of science; on the other hand philosophy and history of science are fields which are themselves of an interdisciplinary nature. In fact, these two aspects are also closely related to each other, as viewing philosophy and history of science as forerunners of an emerging interdisciplinary field may also serve to emphasize the as yet immature character of our understanding of the phenomenon of interdisciplinarity. A Festschrift dedicated to Robert S. Cohen and his interests in the inseparable bonds between the philosophy and history of science provides a natural occasion to deal with both of these aspects: part one of the following essay therefore discusses some features of the development of historical epistemology as an interdisciplinary field, while part two addresses the new technological conditions under which this development takes place today, including some sidelights on the impact of these conditions on the future of interdisciplinary research in general. ${ }^{2}$

1 See Cohen 1985, pp. 204-205.

2 The following sketch of the project of an historical epistemology is largely based on discussions over the last years with Peter Damerow, Gideon Freudenthal, Wolfgang Lefèvre, Peter McLaughlin and other members of a research colloquium based at the Max-Planck-Institute for Human Development and Educational Research in Berlin; see, e. g., Damerow, Freudenthal, McLaughlin, Renn 1991. 
Since the emergence of scientific disciplines is a process involving both social and cognitive factors, only an historical theory of scientific cognition which comprises both the social and the cognitive structures of science will be able to cope with the challenge to our understanding of science that is created by its growing interdisciplinary character. Such a theory, which I would like to call "historical epistemology" following a suggestion by Marx Wartofsky ${ }^{3}$ - exists, however, if at all, only in very rudimentary forms. In the following I will first indicate some of the difficulties associated with the emergence of such a theory and then attempt to outline some of its general features, both on the basis of an analogy with evolutionary biology.

Although it has by now become customary to speak of the "philosophy and history of science" as if they were already one field, the ordinary practice of research and teaching is still far from corresponding to this nominal unification. In spite of the efforts of Cohen and others (in the Boston Colloquium and in the Boston Studies, to give just two examples), and in spite of pathbreaking examples for combined historical and philosophical studies (among the earliest those of Feyerabend, Holton, Kuhn, and Lakatos), the dichotomy between analysis and description, between a philosophy of science that emphasizes ahistoric methodology and metaphysics, and a history of science that is focused on an account of particulars rather than on the explanation of general structures, continues to dominate the academic reality of these fields. It is hence by no means obvious that philosophy and history of science are ready to give rise to an historical epistemology which would need not only to add the models and instruments of the social and cognitive sciences to the traditional methodological arsenal of the philosophy and history of science, but also seek a theoretical coherency that goes beyond exploiting historical case studies in order to flesh out preconceived philosophical opinions. This account of the present situation may serve to illustrate how urgent it is to seriously acknowledge the interdisciplinary character of the task to build an historic theory of scientific knowledge.

3 See Wartofsky 1985. 
Obviously, however, a well-posed question does not guarantee a satisfactory answer, although the realization of the interdisciplinary character of a question may well be a necessary precondition for searching answers beyond longstanding disciplinary entrenchments. But are there any indications that such a search may be successful in the case of historical epistemology? In the following, I will attempt to answer this question by an historical analogy. The present situation of science studies seems to be comparable to that of biology before the advent of Darwin's theory of evolution. In fact, just as science studies today, biology in the first half of the nineteenth century was split into a variety of mutually unrelated subdisciplines, from botany to zoology, from morphology to paleontology. Darwin's theory of evolution radically changed this situation by making it possible to establish systematic conceptual links between "contextual" factors such as the geographical distribution of the species and "internalistic" factors such as the structures described by morphology. 4

From an analogy between pre-Darwinian biology and present-day science studies we can first of all learn that the opposition between contextualist and internalist approaches in the philosophy and history of science may possibly be a consequence only of our lack of understanding of the mutual relationship between external and internal factors. But the analogy may not only help to provide a diagnosis but perhaps also suggest a therapy for improving our understanding of this complex relationship. This hope has its basis in the fact that the analogy does not just refer in general to a process of conceptual integration starting from a variety of heterogeneous subdisciplines but that it compares quite specifically theories of development, biological on the one hand, cognitive and social on the other. In fact, at least this much seems to be certain: if it should be possible at all to formulate a theory of scientific thinking in its interdependence with other areas of human culture, then it must be a developmental theory which does not separate scientific from non-scientific thinking in any absolute way, but which deals with the emergence of scientific thinking within its cultural and social contexts. It is only in this perspective that the tensions between the philosophy and

4 The analogy between historical epistemology and evolutionary biology sketched in the following is based on the historical assessment given of Darwin's discovery in Lefèvre 1984. 
history of science, between internalism and contextualism, between the essence and the appearance of science, between its rational and its irrational moments can be conceivably addressed within a single theoretical framework.

Let me now turn to a closer examination of the analogy between a theory of the development of scientific thinking and Darwin's account of the evolution of biological species: which were the conditions that enabled Darwin to forge a conceptual unity out of a bundle of mutually unrelated pursuits? The historization of nature that is the hall-mark of Darwin's achievement was in part based on an experiential base that did not have an obvious bearing on the question of the historical development of species, namely the practice of breeding. Nevertheless, this practice did supply Darwin with controllable experiences on the change of biological life forms which he could exploit for a theory in which not man but nature herself was to take on the role of the breeder. There is indeed a comparable experiential base that can be made fruitful for a developmental theory of scientific thinking in a way similar to Darwin's use of breeding for an understanding of biological development: studies of cognition in the widest possible sense, including psychology, educational research, and cognitive science.

This large field of study offers both a wealth of empirical knowledge as well as a pool of theoretical models which can be brought to fruition on a historical theory of scientific thinking. That the full potential of this field for the creation of an historical epistemology has so far not been realized may be due to the fact that it is not sufficient to simply transfer the results of laboratory studies into the field of history. Just as it was the case during the emergence of the biological theory of evolution, it is rather necessary to elaborate a genuinely historical theory that will necessarily challenge also many of the theoretical presuppositions implicit in the laboratory studies.

Although the analogy with evolutionary biology does certainly not provide a blue-print for an historical epistemology, it may nevertheless help to formulate some of the theoretical questions to be answered by such an approach. For instance, in both cases mechanisms ensuring the continuity of the development as well as its innovative powers are required. An explanation of continuity in the development of scientific thinking is suggested by noticing 
that what hereditary transmission is to biology, tradition, and in particular the transmission of artifacts from one generation to the next, is to cultural evolution, comprising the history of thinking.

If the transmission of the material artifacts involved in scientific thinking processes - understood in a wide sense so as to include language, scientific formalisms and other means of external representation - forms indeed the back-bone of the social accumulation and tradition of knowledge, then this development is necessarily a self-referential and thus irrevocably historical process in the same sense that biological development is. In other words, in both cases we are not dealing with the autonomous development of structures (life forms, forms of thinking) within an invariable external context (the physical environment, nature) but within a context that is itself defined by the development of living or cognitive structures, as the case might be (natural resources are shaped by the biological development, our experience of nature as it is perceived by science is mediated by material artifacts already endowed with cognitive content).

In biology, the explanation of structural innovation and hence the explanation of the discontinuous aspects of development refer to a steady and by itself continuous process of the exploration of new life resources by the living organisms. In the history of scientific thinking, a similar explanation is required which replaces the still widely accepted but essentially empty idea of the "spontaneous generation" of new concepts. The steady exploration of cultural resources, and in particular of the horizon of possibilities offered by the instruments of science as they are available in a given historical moment, should represent an analogous process allowing to explain the emergence of the new in scientific thinking.

In fact, the instruments of science - in the quite general sense introduced above, that is, including any form of external representation of thinking - appear to offer a suitable basis for such an explanation as they open up - by their very nature as parts of the material world - a range of applications that is wider than that for which they were originally intended. In other words, the knowledge that can be acquired by the application of material means exceeds the cognitive presuppositions necessary for their creation 
(Hegel's List der Vernunft). While the production and tradition of these means can be studied using the traditional methods of the historical sciences, the analysis of the cognitive processes corresponding to the exploration of the range of possibilities determined by these means should refer, as I have pointed out above, to laboratory studies in a similar way as the formulation of evolutionary biology had to rely on the study of changing life forms in breeding.

2.

The discussion in part one has emphasized the need for integrating various disciplines in order to prepare the ground for an historical epistemology. However, the question remains as to how such an integration can actually be accomplished, since it presupposes mastering an enormous array of knowledge that is presently divided into disciplinary structures that may well not correspond to the intellectual structures emerging from a synthesis of this knowledge. It may, for instance, be relevant to such a synthesis to systematically compare - with the theoretical questions of an historical epistemology in mind - historical developments in physics, biology, and chemistry from early modern times until today - but who could actually oversee a quantity of knowledge that presently has to be subdivided into chunks each corresponding to an entire academic career?

I would like to address this question by way of a brief reflection on the historical development of the disciplinary structure of science, in order to examine whether perhaps some of the conditions of this development have changed or are changing in such a way as to create more realistic conditions for the synthesis mentioned above. The disciplinary organization of science as we know it today is the result of an historical development the onset of which can be roughly dated to the end of the eighteenth century. This development depended both on external conditions such as the changing relationship between science and industrial production and internal conditions such as the rapid accumulation of knowledge and the stabilization of areaspecific knowledge structures. Furthermore, the emergence of disciplines would have been impossible without the social mechanisms mediating between the individual and the social 
aspects of knowledge systems, such as the educational system and scientific journals. While the genesis of disciplines enhanced scientific productivity in an unprecedented way, the negative aspects of disciplinary specialization can be recognized already in the nineteenth century. They are visible in the increasing difficulty to communicate knowledge across disciplinary borders and in the failure of attempts to create a scientific world view integrating the knowledge produced by the various disciplines.

It now turns out, however, that precisely on the level of the mechanisms which mediate between the individual and the social aspects of knowledge systems, recent technical developments of information processing have created the preconditions for new forms of the integration of knowledge. The electronic representation of knowledge - for instance in the form of electronic texts - opens up quantitatively and qualitatively new possibilities of access to knowledge which will in the long run also change the social structure of its organization and hence the disciplinary structure of science. They thus provide a case in point for the role of scientific instruments emphasized in part one. In fact, if human thinking is conceived as a process to which the relationship between mental structures and their culturally determined external representations is crucial, then it becomes clear that the introduction of electronic information processing may affect the very nature of scientific thinking processes.

Some of these implications can be strikingly illustrated by referring to the Perseus Project of Crane and collaborators. ${ }^{5}$ By making not only texts of Greek literature electronically available but also images of vases, of archeological sites, maps etc. it furthers the interdisciplinary study of Greek culture in a variety of ways. First of all, the same electronic corpus of material provides access to documents which are still primarily evaluated by different disciplines, such as philology, archeology, or anthropology. Secondly, the electronic access enables a scholar to gain an overview over a much larger body of material in a much shorter time than it has hitherto been possible. In other words, the size of a "digestible unit" of information increases dramatically, and hence for instance the time it takes to test a working hypothesis on a large body of material. But perhaps

5 See Crane 1991 for penetrating reflections on the impact of the new technologies on the humanities. 
the most incisive innovation if compared to traditional forms of the representation of knowledge lies in the "active" character of the new forms of access. By transferring certain mental operations such as searching, grammatical analysis, or translation to the computer, problems which traditionally presupposed a technical competence that could only be acquired by a disciplinary training (such as mastery of the grammar of the ancient Greek dialects) now become intellectually accessible also to the non-specialist.

The example of the Perseus Project also demonstrates, however, that the new possibilities created by the technological development can only be used if they are combined with new forms of the intellectual organization of knowledge. In particular, only the integration of the source material into an hypertext environment enables the user to navigate through an otherwise overwhelming amount of information. Whereas a text is an essentially linear representation of non-linear intellectual structures, a hypertext is a representation of such intellectual structures which itself comprises non-linear features, although it may ultimately also be based on the textual representation of thinking. Almost every traditional book contains features of a hypertext, even if still in a rudimentary form. A scholarly edition, for instance, embeds primary sources into a hypertext structure, which is realized by tables of content, headnotes, footnotes, indexes, bibliographies etc.. A more elaborate hypertext structure as it can be realized in an electronic medium not only provides a network of internal and external references to the sources, but it also allows to overcome some of the basic limitations of access to a linear text structure, limitations which become the more severe the larger the body of information.

The revolutionary consequences of realizing hypertext structures in a new medium can best be illustrated for the example of an index. Because an electronic hypertext link is not only a direction sign but also a vehicle, an electronic index can be much more efficiently used as an actual access to the text than its traditional counterpart because now "browsing through an index" becomes a feasible way of exploring a large text body. In fact, by way of an electronic index, any part of a text can now be "linked" to any other, semantically related part of the text. Conceptually even more important, however, is the possibility of constructing much more complex index structures than is presently customary. 
A traditional index has a flat tree structure necessitating a non-semantical ordering - alphabetical ordering - at the top level. Queries by a scholar are, however, as a rule related to semantic structures and must therefore first be mapped, in a rather artificial way, onto this mechanical ordering. An electronic index, on the other hand, may incorporate several levels of a semantic hierarchy and thus directly represent aspects of the conceptual organization of the knowledge to which it provides access.

But let us return to our original question which - in the light of our reflection on the technological conditions of the organization of knowledge - now becomes the question as to how these new technological developments may help to prepare the knowledge base required for the emergence of an historical epistemology. ${ }^{6}$ In fact it seems rather obvious that the new information processing technologies radically change the conditions of access to the sources of the history of science. Traditional history of science still lacks the broad base of standard editions, translations, textbooks etc. that are the heritage of some of the more mature historical disciplines. The creation of electronic archives will help to overcome the difficulties related to the availability of primary material and with them the phase in which history of science used to be a field of idiosyncratic expertise, divided into Galileo studies, Darwin studies, Einstein studies, etc.. In fact, by making a broad array of primary sources easily available to every interested scholar, electronic archives will bridge many of the obstacles on the traditional route from the location of documents in archives to their intellectual evaluation by the scientific community at large. They will hence radically change the nature of the sometimes narrow minded disciplinary specialization within the history of science.

A broader text base is in fact a crucial condition for the development of an historical epistemology, as this approach depends on the evaluation of a larger set of source materials than traditional chronological, biographical, or descriptive accounts. The contextualist approach to the history of science, for instance, confronts the history of science with new qualities of documentary sources, such as visual images, and

6 For a discussion of the technical implementation of electronic archives in the history of science as they are advocated in the following, see Renn, Damerow, and Galluzzi 1992, prepared with the assistance of Antonella Krige, Jochen Schneider, Martin Schreiber, Martin Warnke, and others. 
makes it necessary to take a broader selection of sources into account than it has hitherto been customary. Pursuing questions of the cognitive sciences within the history of science, on the other hand, lays an increased emphasis on the use of primary material such as drafts, sketchy calculations, etc., which can be seen as immediate representations of thinking processes. High quality electronic reproductions of the originals will allow to study also those features of such documents that might have been filtered away in a traditional editorial process.

This shift towards archival raw material is, however, associated with some potential drawbacks of an electronic publication in comparison to, say, a traditional edition. Having to confront electronic reproductions of originals rather than traditionally edited texts requires, it seems, an increased amount of expert knowledge on manuscripts, handwriting, language etc., and possibly also the need for technical knowledge that is usually only available to computer experts. While these features may indeed counteract the potential of electronic archives to further the development of interdisciplinary competence, they can in fact be more than compensated if the new technologies are not just considered to be a new medium for reproducing sources but as a new and powerful tool for structuring and communicating scholarly information, as is illustrated by the example of the Perseus Project.

In exploring these new intellectual possibilities historical epistemology may even play a pioneering role for the impact of the new technologies on the organization of scientific knowledge in general. If electronic media will in the not-too-distant future become the dominating carriers of scientific communications, the question of how to structure scientific knowledge within and across disciplines will determine in a decisive way the use of the benefits of the new technologies. In a sense, the history of scientific disciplines would then return to its point of departure: while the system of scientific disciplines once replaced traditional models of knowledge classification, ${ }^{7}$ classificatory enterprises would then take on again a central role in organizing a pool of information whose internal connectivity will have rendered largely obsolete the classical concept of a scientific discipline.

7 See Stichweh 1984. 
In this context an historical epistemology may contribute its share to the intellectual organization of scientific knowledge, e. g. by identifying the cognitive models shared by different branches of knowledge. As a side-effect, historical epistemology might then also help to definitely reverse the trend of the philosophy of science towards content-independent methodology. This trend, which began with early Neokantianism and continued with analytical philosophy, may be understood as a response also to the disciplinary specialization which shifted the perspective on the unity of science from metaphysics to the realm of methodological reflection. It becomes, however, more and more obvious that it is not in this metatheoretical realm that the problems of a synthesis of scientific knowledge can be solved. As it turns out, these problems are no longer of a purely academic dimension but a matter of the intellectual and practical mastery of nature, a mastery without which the very survival of the human species may be in danger.

To appear in:

Kostas Gavroglu et. al.

Festschrift in Honor of Robert S. Cohen

Boston Studies in Philosophy of Science 
Bibliography

Cohen, Robert S. "Constraints on Science." In A Portrait of Twenty-five Years. Boston Colloquium for the Philosophy of Science 1960-1985, ed. Robert S. Cohen and Marx W.

Wartofsky. 198-205. Dordrecht, Boston, Lancaster: D. Reidel Publishing Company, 1985.

Crane, Gregory. "Composing Culture. The Authoring of an Electronic Text." Current Anthropology $32(3,1991)$.

Damerow, Peter, Freudenthal, Gideon, McLaughlin, Peter, Renn, Jürgen. Exploring the Limits of Preclassical Mechanics. New York: Springer Verlag, 1991.

Lefèvre, Wolfgang. Die Entstehung der biologischen

Evolutionstheorie. Frankfurt/M, Berlin, Wien: Ullstein, 1984.

Renn, Jürgen, Damerow, Peter, and Galluzzi, Paolo. The Galileo Einstein Electronic Archives Project. NSF Grant Proposal, 1992.

Stichweh, Rudolf. Zur Entstehung des modernen Systems wissenschaftlicher Disziplinen. Physik in Deutschland 1740 1890. Frankfurt am Main: Suhrkamp, 1984.

Wartofsky, Marx W. "Perception, Representation, and the Forms of Action: Towards an Historical Epistemology." In $\underline{A}$ Portrait of Twenty-five Years. Boston Colloquium for the Philosophy of Science 1960-1985, ed. Robert S. Cohen and Marx W. Wartofsky. 215-237. Dordrecht, Boston, Lancaster: D. Reidel Publishing Company, 1985. 